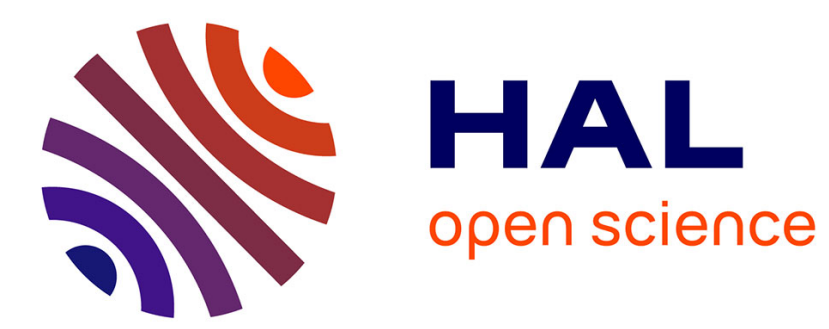

\title{
L'ouverture commerciale de l'Algérie
}

\author{
Mehdi Abbas
}

\section{To cite this version:}

Mehdi Abbas. L'ouverture commerciale de l'Algérie: Apports et limites d'une approche en termes d'économie politique du protectionnisme. Revue Tiers Monde, 2012, 210, pp.51-68. halshs-00717593

\section{HAL Id: halshs-00717593 \\ https://shs.hal.science/halshs-00717593}

Submitted on 13 Jul 2012

HAL is a multi-disciplinary open access archive for the deposit and dissemination of scientific research documents, whether they are published or not. The documents may come from teaching and research institutions in France or abroad, or from public or private research centers.
L'archive ouverte pluridisciplinaire HAL, est destinée au dépôt et à la diffusion de documents scientifiques de niveau recherche, publiés ou non, émanant des établissements d'enseignement et de recherche français ou étrangers, des laboratoires publics ou privés. 


\section{ÉCONOMIE DU DÉVELOPPEMENT DURABLE ET DE L'ÉNERGIE}

\section{L'ouverture commerciale de l'Algérie}

Apports et limites d'une approche en termes D'économie politique du protectionnisme

Mehdi Abbas

Septembre 2011

Cahier de recherche $n^{\circ} 13 / 2011$ 



\section{L'ouverture internationale de l'Algérie. A pports et limites d'une approche en termes d'économie politique du protectionnisme}

M ehdi A bbas, maître de conférences, EDDEN, Université de Grenoble, CNRS.

Septembre 2011

En s'appuyant sur la littérature de l'économie politique de la protection, l'article développe une grille de lecture de l'évolution de la politique d'ouverture de l'A Igérie sur la période 1986-2012. II met en évidence trois facteurs explicatifs de l'ouverture: le contexte macroéconomique, le jeu des intérêts sectoriels des principaux acteurs du système national d'économie politique et le contexte intellectuel. Cela permet de distinguer trois périodes caractéristiques de la politique commerciale algérienne: la déligitimation de la protection (1986-1994) ; I'ouverture obligée sous contrainte des institutions internationales (1994-1998) et la recherche d'une maîtrise de l'ouverture (1999-2011).

M ots clés : protection, économie politique de l'ouverture, conflit distributif.

\section{Algeria's International Openness. The contribution of Political Economy of Protection Approaches}

Using the literature pertaining to the political economy of protection, this paper develops an analysis of the A Igerian openness strategy over 1986-2012 period. It puts forward three main factors: the macroeconomic context, the sectoral interests of the main actors of the national political economy system and the intellectual context. This allows to distinguish three different periods in the Algerian trade policy: the deligitimation of protection (1986-1994), the bounded openness under the constraint imposed by international organizations (19941998) and the look for managed openness (1999-2011).

Key words : protection, political economy of openness, distributive conflict. 
Les réformes affectant le commerce extérieur de l'A Igérie constituent un élément important du processus de transformation engagé par le pays à partir de la seconde moitié des années 1980. L'objet de cette contribution est de fournir un cadre analytique de l'évolution de la politique d'ouverture de I'Algérie en s'appuyant sur les problématisations et conceptualisations de l'économie politique du protectionnisme (EPP).

Conformément à l'objectif du dossier, notre contribution se propose de dresser un bilan des transformations survenues dans le rapport au monde de l'économie algérienne durant les années 1980-2010 et d'apporter des éclairages sur les orientations en cours. II n'est pas question d'analyser la pertinence ou non des choix de politique commerciale faits par les gouvernements al gériens successifs, ni d'établir, à travers une analyse normative, les pertes et les gains pour l'économie nationale ou pour l'une de ses composantes suite à ces choix, encore moins d'indiquer quelle serait la politique commerciale la mieux à même d'assurer le développement ou la diversification productive de l'A lgérie.

Le point de départ de l'analyse est double. D'une part, le travail de Hall (1997) concernant les trois déterminants des évolutions des politiques économiques servira de grille de lecture des évolutions de la politique commerciale algérienne. D'autre part, la proposition de Gilpin (1987) selon laquelle l'ouverture possède une dimension structurelle (les avantages comparatifs, les prix relatifs, etc.), stratégique (les interactions entre acteurs) mais également institutionnelle, qui renvoie aux caractéristiques du "système national d'économie politique » (SNEP). Nous reprenons cette notion mais en en proposant une définition élargie puisqu'il s'agit de l'ensemble des relations complexes, contradictoires et hiérarchisées unissant les différents individus, groupes et groupes de groupes qui, d'un côté, utilisent leurs positions et les différents appareils de l'Etat en vue d'imposer leurs préférences et intérêts et, de l'autre, ont accès par des canaux légaux ou non, formels ou non, aux ressources de l'Etat. Celles-ci sont d'autant plus importantes que l'Etat algérien présente les propriétés institutionnelles, économiques et politiques d'un Etat rentier (Luciani, 1987). Celui-ci est, au travers de ses agences, administrations, gouvernement et ressources dont il dispose, un acteur central du SNEP ${ }^{1}$.

Ces éléments étant posés, il est possible de mettre en évidence, sur la période 1986-2012, un biais étatique dans la formulation et la gestion de l'articulation national-international. Ce biais est déterminé par la combinaison de trois séries de facteurs : le contexte macroéconomique, le jeu des intérêts sectoriels des différentes composantes du SNEP algérien et le contexte intellectuel dans lequel s'élabore la politique d'ouverture. Ce biais est dû au fait que les procédures concrètes d'internationalisation de l'économie algérienne participent des dispositifs d'exercice et de légitimation du pouvoir. En effet, Le rapport au marché mondial intermédié par l'Etat - influence la dynamique structurelle et institutionnelle du marché national et détermine la légitimité du régime politique (Smith, 2006 ; Schwartz, 1994).

Cette contribution s'organise en deux parties. La première présente les approches de l'EPP afin de faire ressortir les principaux déterminants des choix de politique commerciale figurant dans la littérature. Elle se prolonge par une contextual isation de l'analyse au cas al gérien. Les spécificités du rapport au monde de l'économie al gérienne ayant été établies, la seconde partie

\footnotetext{
${ }^{1}$ En portant notre attention aux compromis au sein du SNEP nous nous inscrivons dans le sillage de ce que $\mathrm{K}$ han (1995) nomme le "political settlement» (l'équilibre de pouvoir entre classes et groupes affectés par les institutions). De même, le conflit distributif lié à l'ouverture fait partie des « coûts de transition » identifiés par I'auteur. Nous nous en éloignons en proposant une lecture qui ne se réduit pas aux facteurs domestiques.
} 
rend compte des évolutions de la politique d'ouverture de l'A Igérie. Elle se termine par une conclusion récapitulant les principales propositions de l'anal yse.

\section{Une économie politique des choix de politique commerciale appliquée à l'Algérie}

L'EPP s'intéresse aux conditions socio-économiques et sociopolitiques qui président aux choix économiques entre ouverture et protection, à la fois d'un point de vue global et sectoriel. Elle a pour point de départ un refus de poser que seule la rationalité des avantages comparatifs, c'est-à-dire l'adaptation au système de prix relatifs, prévaut dans la définition du contenu et des modalités de la politique commerciale (Gourevitch, 1986 ; Hillman, 1989). La littérature admet que le choix de l'ouverture résulte de la combinaison de trois séries de facteurs: le contexte intellectuel, le jeu des intérêts d'acteurs et le contexte macroéconomique. Cette première partie montre en quoi ils structurent le SNEP algérien.

\section{Le contexte intellectuel}

L'une des hypothèses de $\mathrm{H}$ all est que la dynamique des idées permet à la fois une justification et une légitimation des préférences et choix de politique commerciale. D'ailleurs, l'importance du contexte intellectuel constitue un des apports des approches constructivistes de la politique commerciale (Sikkink, 1991 ; Goldstein, Keohane, 1993). L'ouverture y est analysée comme une construction idéologique d'un problème de développement économique qui conditionne les objectifs et les instruments que se donne le décideur en matière d'insertion internationale. La politique commerciale ne dépend pas uniquement de l'organisation sectorielle de l'économie (la structure des avantages comparatifs) et de la concentration des groupes d'intérêt mais également de la cohésion doctrinale des individus formant le groupe des décideurs politiques (Destler, Odell, 1987).

Ainsi, I'orientation protectionniste de la période 1965-1988 correspond à la stratégie de développement de l'A Igérie ${ }^{2}$, le régime de monopole du commerce extérieur accompagnant la planification centralisée et l'étatisation de l'activité économique. La politique de réforme du commerce extérieur se devait tout d'abord de dé-légitimer l'orientation protectionniste de la période précédente. Ainsi, durant la première moitié des années 1980, dans le cadre de " l'enrichissement de la charte nationale », une nouvelle rhétorique s' est développée, appelant à une « modernisation de l'économie » $\mathrm{s}^{\prime}$ appuyant sur des « normes définies mondialement » et obéissant aux « lois universelles de la logique économique» (B rahimi, 1991; Henni 1991). La nouvelle charte nationale, adoptée le 16 janvier 1986, constitue une étape significative dans la réorientation doctrinale de la stratégie d'ouverture. Cela d'autant plus qu' elle s'inscrit dans les transformatiosn systémiques à l'œuvre dans l'économie internationale. A insi, sous couvert de "crise du commerce extérieur » (Goumeziane, 1994), I'A Igérie s' engage dans la vague libre-échangiste de la seconde moitié des années 1980 soulignée par Rodrik (1995b) et impul sée par les institutions financières internationales.

\footnotetext{
${ }^{2}$ La politique d'exportation et de valorisation des hydrocarbures devait permettre l'élargissement des bases internes d'accumulation en vue d'une plus grande autonomie économique et politique. Cependant, cette stratégie n'a pas permis de réduire la dépendance aux importations de produits alimentaires, de produits semi-finis et d'équipement (Ecrement, 1986; Benbitour, 1992). Cette problématique est toujours présente puisque la dépendance sans cesse croissante aux importations, particulièrement depuis 2004, est posée comme l'enjeu central de l'insertion internationale du pays du fait que $70 \%$ des financements du plan (2006-2014) ont été consacrés aux importations.
} 
L'évolution du contexte intellectuel accompagne l'apparition d'une configuration politique nouvelle qui trouve ses racines dans le changement survenu au sommet de l'Etat fin des années 1970. Elle lui offre un moyen de légitimer ses nouvelles orientations de politique économique. Cela nous conduit à traiter des intérêts d'acteurs car le choix de l'ouverture participe aux recompositions économiques et politiques au sein du SNEP.

\section{Le jeu des intérêts factoriels et sectoriels}

En s'écartant des déterminations exogènes du protectionnisme, l'EPP introduit les comportements du pouvoir politique et son rapport aux groupes d'intérêt. La politique commerciale - envisagée comme un marchandage entre une demande et une offre de protection - dépend en grande partie du jeu des acteurs et de leur stratégie de lobbying sur une base factorielle ou sectorielle (Rodrik, 1995a ; B aldwin, 1996).

Dans le cas de l'Algérie où le secteur des hydrocarbures ne rencontre aucune contrainte internationale et constitue le seul secteur d'exportation du pays ( $98 \%$ des exportations totales en 2010), la maîtrise des importations (flux, institutions et configuration des acteurs) constitue l'enjeu essentiel de l'économie politique de l'insertion internationale compte tenu de ses effets sur le système productif national et des situations de rente que génèrent les arbitrages étatiques en la matière.

Loin de se caractériser par une régulation concurrentielle et impersonnelle correspondant à I'approche factorielle de I'EPP, le SNEP algérien correspond à un schéma où le conflit distributif entre groupes d'intérêt est structurant ${ }^{3}$. II explique les différences de niveaux de protection d' un secteur à un autre dont profitent les groupes d'intérêt. De même, l'importance des mesures non tarifaires (tableau 1) est la manifestation d'un jeu d'acteurs visant à minimiser les effets transformationnels associés à l'ouverture (Baldwin, 1989) et reflètent une régulation clientéliste, généralement contingente à une économie pétrolière rentière.

\footnotetext{
${ }^{3}$ On sous-estime l'ampleur du conflit distributif prévalant en Algérie, mal reflété par la seule mesure des inégalités de revenus, elles-mêmes très mal mesurées en raison d'insuffisances statistiques. Les violences régionales et urbaines recouvrent la plupart du temps des conflits autour de l'accès aux dividendes de la rente.
} 

Tableau 1 : Indicateurs de restrictions commerciales 1995-2009 de l'Algérie et
comparaison avec les pays de la région

\begin{tabular}{|l|c|c|c|}
\cline { 2 - 4 } \multicolumn{1}{l|}{} & Algérie & UM A & ME NA \\
\hline Tariff Trade Restrictiveness Index (TTRI) & 12,97 & 15,74 & 13,79 \\
\hline M arket A ccess - TTRI & 0,80 & 2,98 & 2,23 \\
\hline Institutional Environment (IE) - Ease of & 133,3 & 123,9 & 125,3 \\
Doing Business & & & \\
\hline Real growth in trade of goods and services & 4,05 & 5,74 & 4,91 \\
& & & \\
\hline
\end{tabular}

Source : Banque mondiale, 2010, World Trade indicators. Trade-at-a-Glance, Pour le contenu et la méthodologie des indicateurs, on peut consulter : http://www.worl dbank.org/wti

$M$ ais I'analyse sectorielle doit être complétée par une approche institutionnelle de la politique commerciale. En effet, le SNEP algérien ne peut être appréhendé sous l'angle d'une démocratie de marché dans laquelle les acteurs sont en concurrence et où le choix de politique commerciale est le produit du jeu électoral des partis politiques. L'hypothèse de l'électeur médian semble n' être d' aucune portée heuristique tant elle est éloignée de la réal ité du SNEP algérien qui, par ailleurs, $n$ 'assume pas la fonction d'agrégation des préférences, de définition des priorités collectives et de l'intérêt national.

L'approche institutionnelle porte son attention sur I' offre de protection faisant des « courroies de transmission » des demandes sociétales (Goldstein, 1988) et de l'autonomie plus ou moins marquée de la bureaucratie étatique vis-à-vis des groupes socio-économiques (Chang, 1993) un élément de compréhension de la forme et de la substance de la politique commerciale. De fait, les différentes composantes du SNEP sont en situation de dépendance vis-à-vis des arbitrages étatiques. Les acteurs maîtrisant le commerce extérieur ne bénéficient de cette position que grâce à des décisions publiques ${ }^{4}$. Le recours à une approche institutionnelle de la protection s'impose d'autant plus que l'appareil décisionnaire étatique est en son sein traversé par des conflits de préférences et d'intérêts (Dahmani, 1999 ; A ghrout, B ougherira, 2004).

A insi, la période 1986-2011 n'est pas tant déterminée par la problématique libre-échange vs. protection, que par un mouvement contradictoire entre étatisation et désétatisation de la politique du commerce extérieur. Cela conduit à relativiser la libéralisation du commerce

\footnotetext{
${ }^{4}$ Les derniers travaux de North montrent que l'analyse du changement institutionnel bascule des «coûts de transaction » à la « recherche de rentes ». Les comportements de la coalition dominante tiennent un rôle central dans l'attribution des rentes à l'origine de la dynamique économique et politique d'une société ( $N$ orth, Wallis, W eingast, 2010). En revanche, contrairement aux auteurs, notre cadre analytique postule que la gestion du conflit social et distributif est l'élément moteur du comportement des élites.
} 
extérieur dont le taux d'ouverture (figure 2), structurellement élevé dans une économie pétrolière, ne permet pas de saisir sa portée réelle ${ }^{5}$. Ce mouvement est contradictoire car I'appareil de décision étatique, qui en est le principal initiateur, en constitue simultanément la principale source de neutralisation, voire de blocage. La raison principale réside dans la structure conflictuelle des intérêts socio-économiques articulés au pouvoir étatique. $\mathrm{M}$ ais la compréhension de la «boîte noire » de l'E tat pétrolier algérien demeure entière et excède I'objet de cette contribution. L'analyse de l'ouverture doit prendre en compte la capacité de certains acteurs à accéder aux ressources économiques et politiques (aides, protection, information) afin de minimiser les risques de leur exposition à la concurrence internationale. Les stratégies d'adaptation aux décisions publiques génèrent de nouveaux réseaux, qui pèsent sur la trajectoire des réformes non pas en termes de prise de décision, mais par les effets de recomposition non anticipés, voire improvisés, induits par leur adaptation aux nouveaux enjeux de production et de redistribution de richesse.

Figure 2 : Taux d'ouverture en perspective historique

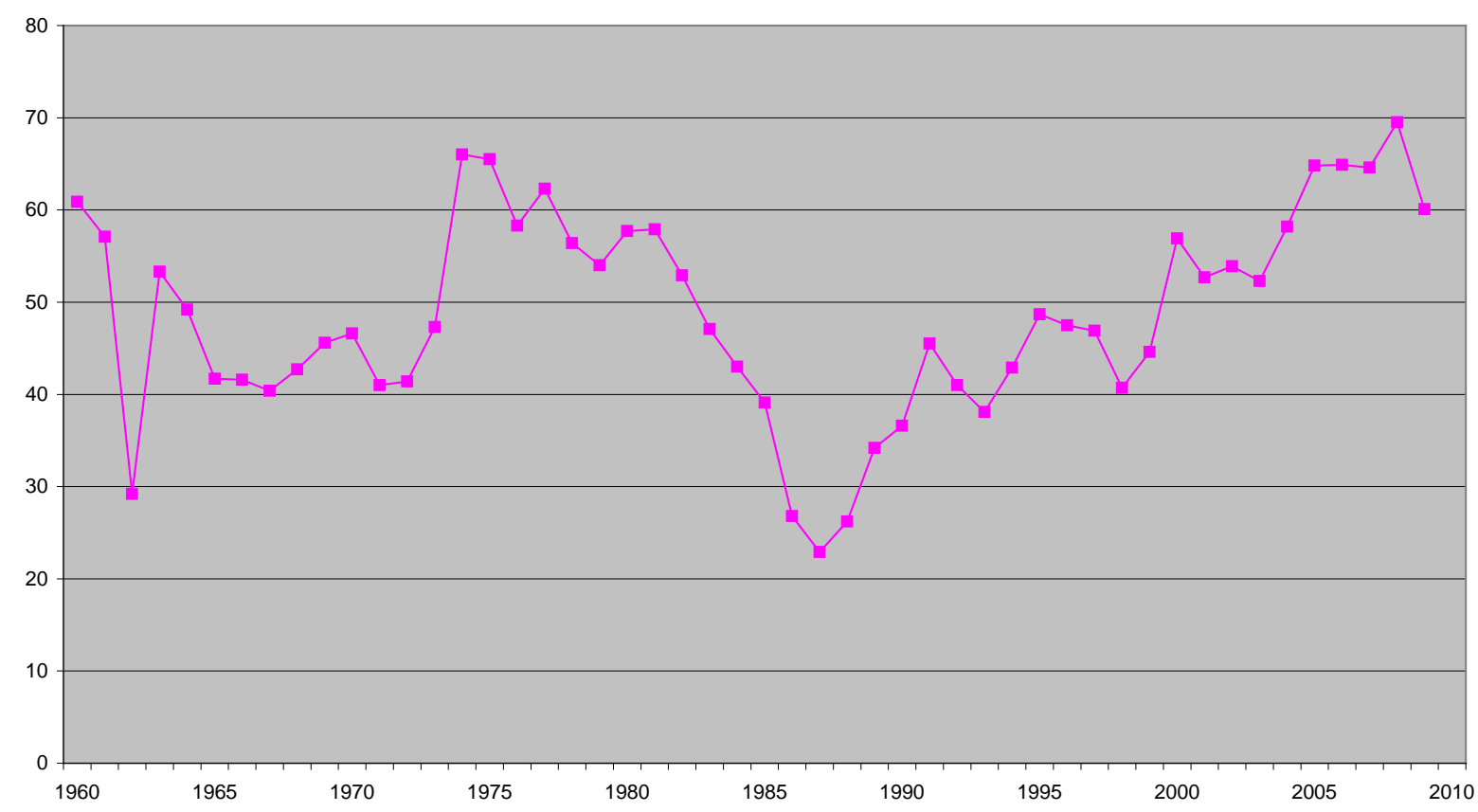

Source: W orld Bank, 2011.

L'approche en termes de SNEP relativise la thèse de "I'Etat bunker » (Henry, 2005) puisque le système est envisagé comme un ensemble de réseaux de pouvoir entretenant des relations hiérarchiques et qui doit intégrer l'hétérogénéité des préférences et des intérêts au sein de I'appareil étatique et gouvernemental, facteur de complexification de la construction d'un consensus (Nelson, 1999). Le jeu des intérêts d'acteurs produit un SNEP dont les propriétés institutionnelles s'articulent au régime de croissance de l'économie algérienne. En effet, l'importance des réseaux d'importation dépend directement des procédures de recyclage des revenus d'exportation d'hydrocarbures. Dès lors, l'analyse des conflits d'intérêt doit être mise en rapport avec le contexte macroéconomique du pays.

\footnotetext{
${ }^{5}$ Outre qu'il ne renseigne ni sur la qualité de l'environnement institutionnel, ni sur la nature des biens importés et exportés, considérer l'indicateur d'ouverture sans porter son attention sur les mesures de contingentement, les licences d'importation, les autorisations administratives, les subventions, etc., peut conduire à une vision biaisée du processus.
} 


\section{Le contexte macroéconomique}

Une troisième explication pointe sur le contexte macroéconomique comme variable orientant la politique commerciale (Cassing, 1991 ; D razen, G rilli, 1993) ou conduisant à l'adoption de réformes du commerce extérieur (K rueger, 1993). Une relation étroite s'établit entre conflits distributifs, contexte macroéconomique et préférence pour le libre-échange (Fernandez, Rodrik, 1991).

L'importance du contexte macroéconomique est liée à la central ité du rapport au monde dans l'économie politique de la croissance al gérienne. Compte tenu de l'importance du secteur des hydrocarbures (pétrole et gaz) en termes de PIB, de revenus d'exportations et de fiscalité (98 $\%$ des recettes d'exportations, $48 \%$ de la valeur ajoutée, $28 \%$ de la richesse nationale et 80 $\%$ des recettes fiscales) (FMI, 2009), I'A I gérie présente les caractéristiques d'une économie pétrolière (Seers, 1964). Par conséquent, l'articulation national-international est I'un des principaux « lieux » où se concrétise la valorisation de la rente, elle est au cœur des conflits distributifs structurant le SNEP (schéma 1).

Dès lors, comme l'a montré Rogowski (1989), un changement des termes de l'échange, des spécialisations et de la composition des échanges internationaux modifie les «alignements politiques domestiques». De même, Garret et Lange (1996) estiment que I'internationalisation d'une économie change la nature du conflit distributif. Mais contrairement à Rogowski, ils insistent sur le fait que l'une des fonctions des institutions domestiques est d'atténuer l'intensité de ce changement, réintroduisant ainsi le rôle du SNEP. Ainsi, l'ouverture a entraîné - processus toujours en cours - une recomposition et un repositionnement des intérêts économiques et politiques en élargissant la sphère privée et en autorisant la présence d'acteurs non résidents. Elle conduit à une redéfinition des procédures d'accès aux ressources de l'E tat et des arrangements formels et informels qui leur sont sousjacents. 
Schéma 1 : Articulation entre l'international et l'économie politique de la croissance

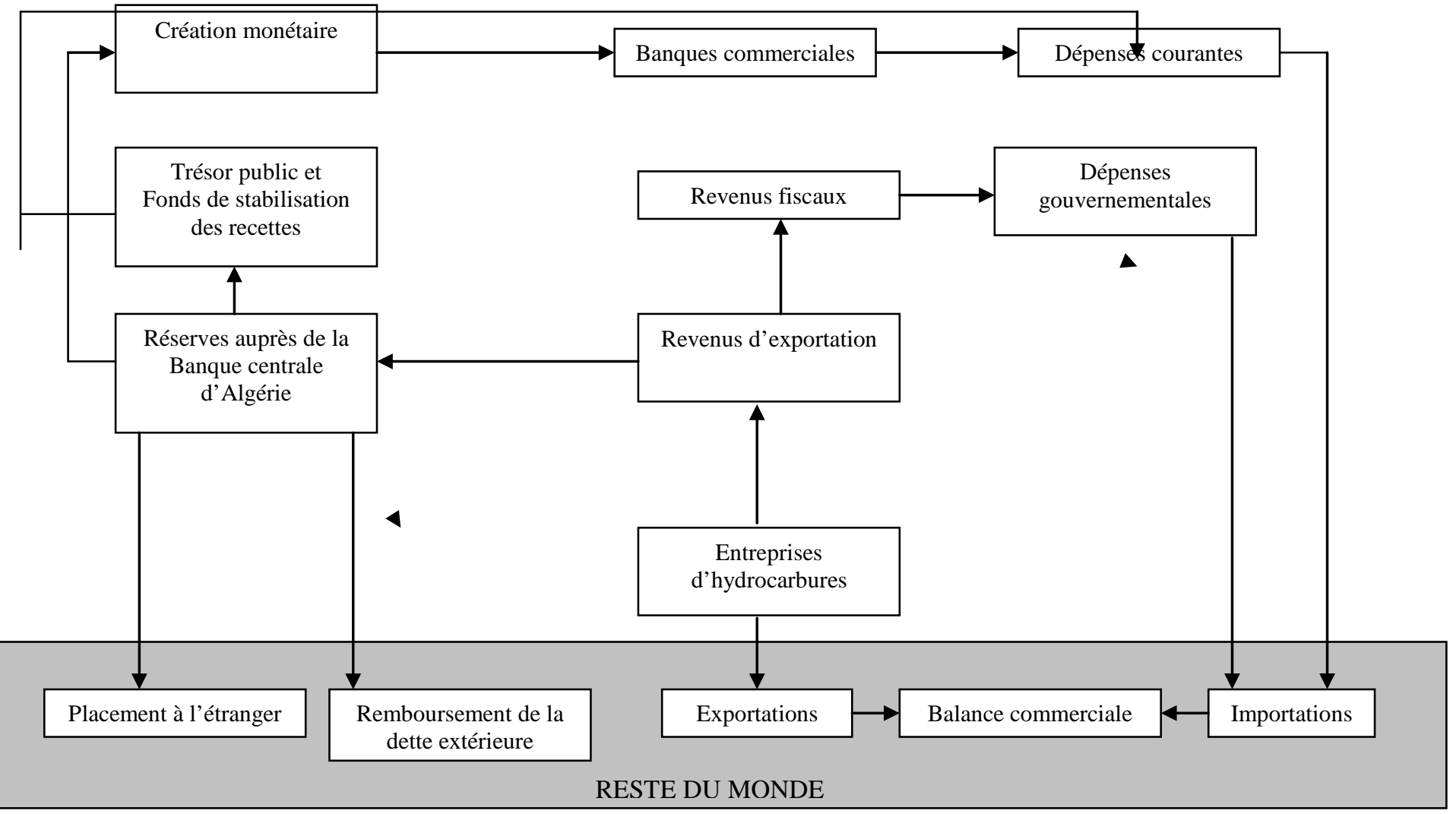

Source: Composition de l'auteur avec adaptation à partir de Aïssaoui (2001) et Talahite, Hammadache (2011b).

Les revenus du secteur des hydrocarbures sont à l'origine du seul revenu d'exportation significatif et des revenus fiscaux que l'Etat utilise afin de mener une politique de croissance tirée par la dépense publique. Par le biais de la Banque centrale, une partie de ces revenus est placée à l'étranger, conditionnant la gestion du taux de change puisque les exportations sont libellées en dollars alors que les importations le sont en fonction d'un panier de monnaies ${ }^{6}$. Les revenus d'exportation servent également de support à la création monétaire qui irrigue l'ensemble de l'économie au travers des crédits distribués par les banques secondaires et le Trésor public. Ces liquidités sont à l'origine de la demande d'importation de biens et services déterminant l'équilibre de la balance des transactions courantes. L'évolution de cette dernière (figure 1) fait ressortir l'absence d'une contrainte extérieure réelle exceptée lors d'épisodes précis (1986-1989 et 1994-1996) durant lesquels les autorités ont été amenées à « réformer » les modalités économiques de contrôle du SNEP.

\footnotetext{
${ }^{6}$ Les principaux partenaires commerciaux de l'A Igérie en termes d'importations sont la France (20\%), I'Italie (11 \%), la Chine (9\%), I'Espagne (8\%), I'A llemagne (5\%). En termes d'exportations ce sont les Etats-U nis (24 $\%)$, I'I talie (15\%), I'Espagne (11 \%), le Canada (10\%), la France (9\%) (B anque mondiale, 2010).
} 


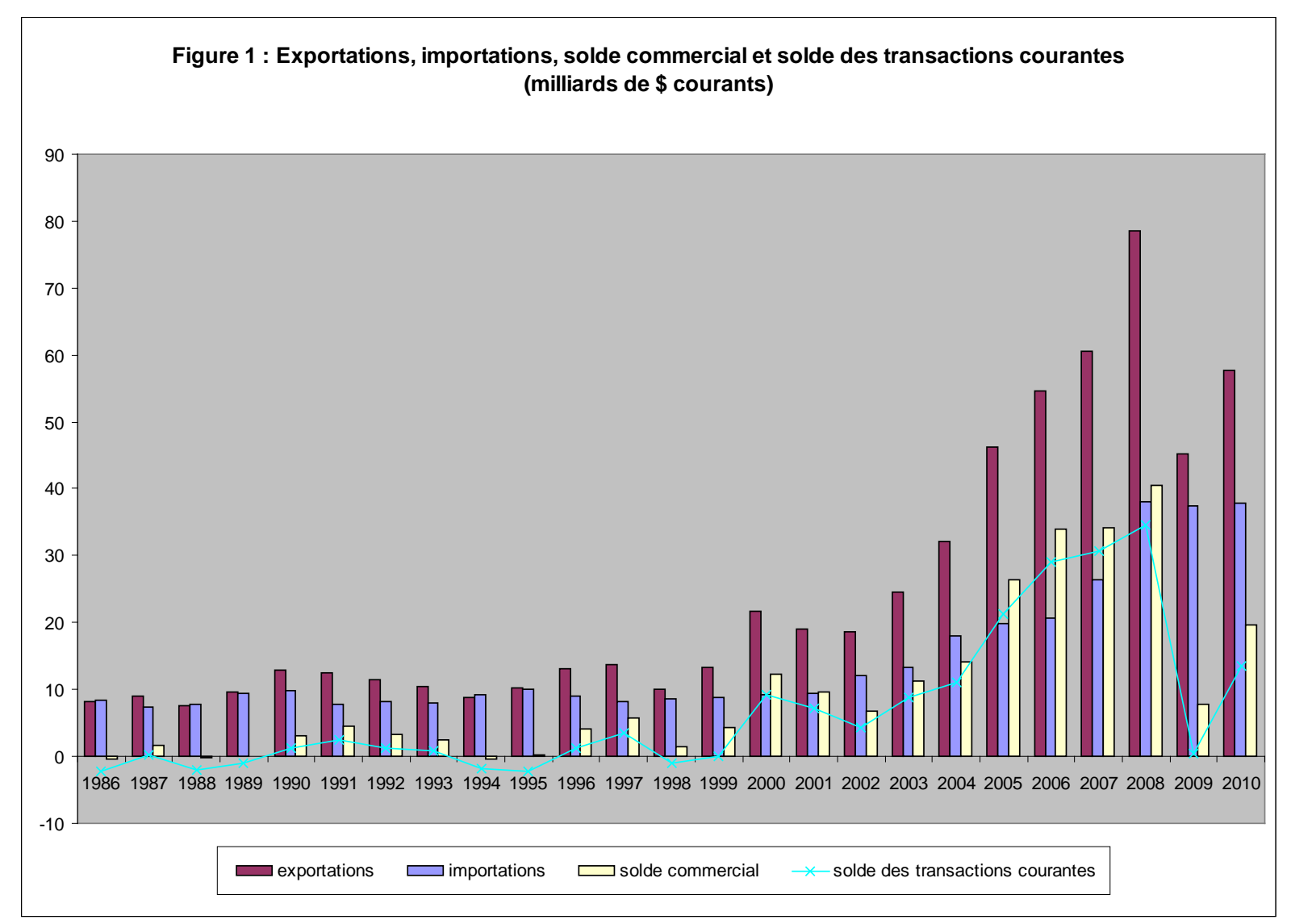

Source: IM F.

L'objet de cette partie était de faire ressortir la pluralité des déterminants tant des formes que de la substance de l'ouverture dans le cas de l'A I gérie. La seconde partie périodise le rapport au marché mondial au prisme de la combinaison des trois séries de facteurs mis en évidence.

\section{M ise en perspective analytique de l'ouverture internationale de l'A lgérie}

Entre 1986 et 2010, la gestion de l'articulation national/international a connu trois périodes.

\section{6-1994. La dé-légitimation du protectionnisme}

Le réel changement survient avec la dégradation du contexte macroéconomique lié au retournement du marché pétrolier en 1986, qui fait chuter les revenus d'exportation de $40 \%$ et accroît les échéances de la dette extérieure (Bouyacoub, 1997). L'équipe d'experts réunie autour du secrétaire général de la Présidence M ouloud Hamrouche propose une vision à long terme basée sur la recherche d'une insertion active dans l'économie mondiale (Corm, 1993), sous-entendu que, jusqu'à présent, la stratégie de dével oppement n'a pas permis cela.

Le dépôt de candidature en vue de l'accession au GATT (1987) constitue la première manifestation de ce changement du contexte intellectuel et macroéconomique. Elle vise trois objectifs: i) la diversification des échanges afin de sortir d'une spécialisation étroite, la libéralisation du commerce extérieur constituant la condition première de cette diversification à moyen-long terme des exportations et de la production ; ii) le relèvement du niveau général de la compétitivité industrielle afin de mieux préparer les industries à affronter les effets de la 
concurrence internationale et iii) la maîtrise et le contrôle des importations des produits alimentaires. Le dispositif législatif promulgué en juillet 1988, même s'il modifie peu l'organisation antérieure du commerce extérieur, introduit le principe selon lequel I'Etat exerce désormais le monopole sur le commerce extérieur par le biais de concessions accordées à des entreprises et organismes publics et à des groupements d'intérêt commun. Mais la pénurie de change dans laquelle se trouve I'Algérie contribue à annihiler cette première désétatisation du commerce extérieur.

Début 1989 une nouvelle constitution, qui lève officiellement les barrières à la libéralisation économique, est adoptée alors qu'à la fin de la même année le courant dit des «réformateurs» arrive aux affaires, M. Hamrouche accédant au poste de chef de gouvernement (septembre 1989-juin 1991). S'enclenche alors un mouvement de désétatisation du commerce extérieur avec la loi de finances complémentaire de 1990 qui admet désormais l'établissement de firmes nationales ou étrangères d'importation et $d^{\prime}$ exportation ${ }^{7}$. Cette première désétatisation du commerce extérieur s'inscrit dans le cadre des accords de stand-by négociés et signés avec le FMI (mai 1989 et juin 1990), constituant également une mesure stratégique de gestion du rapport avec l'institution financière internationale (Benissad, 1991).

Cette libéralisation demeure toutefois limitée en raison de l'impossibilité de disposer de concours bancaires pour le financement des opérations de commerce extérieur, de la nécessité de ne traiter, de n'acheter et vendre que les produits expressément autorisés, et de l'obligation faite aux concessionnaires non résidents de s'engager à produire les biens localement (B enissad, 1997). M ais le plus important est que cette loi modifie le jeu des intérêts d'acteurs en autorisant les concessionnaires et les grossistes agréés à importer des produits de consommation, des biens d'équipement et des biens de consommation industriels pour les revendre en l'état. Elle est à l'origine de l'émergence d'un nouveau groupe socio-économique concentré autour des activités d'import et d'export, que nous qualifions de " commercialiste » au lieu de «nouveaux entrepreneurs » tels qu'ils sont parfois nommés ${ }^{8}$.

A la chute du gouvernement Hamrouche, un mouvement de re-étatisation du commerce extérieur s'amorce. II culminera avec le gouvernement de B elaïd A bdesslam (juillet 1992octobre 1993) qui promulgue en août 1992 une instruction instaurant un «comité ad hoc interministériel chargé du suivi du commerce extérieur et de la nature des engagements commerciaux avec l'extérieur » chargé de fixer, en relation avec le système bancaire, les normes d'accès aux devises et aux crédits pour les importateurs. A nouveau, selon le gouvernement, la rareté des ressources en devises (contexte macroéconomique) exige ce type de mesure.

\footnotetext{
${ }^{7}$ Une réforme tarifaire est mise en œuvre en 1992 avec l'adoption de la nomenclature du système harmonisé. La structure tarifaire passe de 18 à 7 taux et le taux maximal ramené de 120 à $60 \%$. Un nouveau code de l'investissement extrêmement incitatif à l'IDE est adopté en 1993.

${ }^{8}$ Cela rejoint la constatation de B ouyacoub (1997) pour qui «l'économie de marché en Algérie, avant d'être un modèle d'organisation économique, s'est d'abord imposée à travers le secteur commercial ». Le bulletin d'information et de statistiques du ministère de la PME et de l'Artisanat indique que, pour I'année 2009, les 18153 importateurs privés, sur les 19469 que compte le pays, sont à l'origine de 79,25\% du montant global des importations. V oir http://www.pmeart-dz.org/fr/bulletin_2009.php
} 
Période de profonde instabilité économique mais surtout politique, la fin des années 1980 et le début des années 1990 sont marqués par une dégradation du contexte macroéconomique et par un changement nécessairement conflictuel d'orientation idéologique au sein de l'appareil d'Etat. La détérioration continue de la situation financière conduit le gouvernement de Rédha M alek (août 1993-avril 1994) à négocier un programme de stabilisation avec le FM I, ouvrant ainsi sur une nouvelle période en terme d' articulation entre le national et l'international.

\section{4-1998. L'ajustement structurel et l'ouverture contrainte}

Cette période débute lorsque l'A Igérie, en situation de quasi-cessation de paiements, accepte les termes d'un plan d'ajustement structurel (PAS) signé en avril 1994. La mise en œuvre du plan demeure partielle et sélective en raison du contexte politique et sécuritaire. L'Algérie obtient à ce titre un ensemble de mesures dérogatoires. Le PAS s'articule autour de quatre grands objectifs : i) favoriser une forte croissance économique; ii) assurer une convergence rapide de l'inflation vers les taux en vigueur dans les pays industrialisés; iii) atténuer les retombées transitoires de l'ajustement structurel sur les couches les plus défavorisées de la population et iv) rétablir la viabilité de la position extérieure tout en constituant des réserves de change suffisantes (IM F, 1998) ${ }^{9}$. A ce titre, il prévoit une ouverture accrue de l'économie algérienne et sert de catalyseur aux processus d'ouverture multilatérale et bilatérale.

Ainsi l'Algérie est amenée, en 1996, à réactiver le dossier de I'accession à la nouvelle Organisation mondiale du commerce créée l'année précédente (A bbas, 2009). Cette création comporte deux innovations majeures dont les conséquences ont été sous-estimées par les décideurs algériens de l'époque. La première concerne l'engagement unique qui amplifie la contrainte adaptative au nouveau régime commercial multilatéral. La seconde est l'obligation de réciprocité des traitements douaniers privilégiés. Les pays en développement - dont I'Algérie - qui bénéficiaient d'une franchise douanière pour leurs exportations vers les pays industrial isés depuis les années 1970 doivent désormais accepter la création de zones de libreéchange pour pouvoir continuer à bénéficier de cet avantage. Cela conduit les autorités algériennes à ouvrir des négociations avec l'U nion européenne dans le cadre du processus de Barcelone (1995) dont l'objectif est d'aboutir à une zone de libre-échange (2012) au travers de l'accord d' association signé en 2002.

Pour le SNEP I'ouverture s' apparente désormais à une contrainte imposée par les institutions internationales ( $\mathrm{UE}, \mathrm{FMI}, \mathrm{OMC}$ ), ce que certaines franges du système instrumentalisent en usant du référant nationaliste pour dénoncer des pertes d'autonomie économique et réglementaire. Toutefois, la coalition dirigeante engage certes un processus de désétatisation en raison des contraintes financières mais également parce qu'il lui permet sa légitimation, par le biais des négociations internationales bilatérales (UE) et multilatérales (FM I et OMC), confirmant que l'ouverture constitue une procédure de pérennisation de ce que Werenfels (2007) nomme la «politically relevant elite». Elle produit également une pluralisation des intérêts offrant de nouvelles opportunités d'accumulation à des acteurs jusqu'alors exclus et conduisant à une recomposition économique et politique. Cette seconde période achève de

\footnotetext{
${ }^{9}$ Le PAS comportait à titre indicatif : libéral isation de produits dont l'importation avait été soumise à des critères techniques et professionnels, élimination de toutes les interdictions d'exportation, libéralisation des importations de matériel professionnel et industriel, élimination de l'obligation faite aux importateurs de certains produits de respecter des critères professionnels et techniques, réduction du taux maximum des droits de douane de 60 à 50 $\%$ (1996) puis de 50 à $45 \%$ (1997), convertibilité du dinar algérien pour les transactions extérieures courantes, I'A Igérie acceptant les obligations imposées par l'article V III des statuts du FM I (1997).
} 
marginaliser les «industrialistes» au profit des «financiers» et consolide la position des « commercialistes» au sein du SNEP.

\section{Depuis 1999. La recherche d'une maîtrise de l'ouverture}

La troisième période caractéristique de la politique d'ouverture débute au moment où commence un nouveau cycle politique avec l'élection présidentielle d'avril 1999 qui voit la victoire d'Abdelaziz Bouteflika. Elle s'ouvre avec la fin du programme d'ajustement structurel (mai 1998) et le retour aux équilibres macroéconomiques et financiers contribuant à orienter l'agenda post-ajustement vers I'amélioration des déterminants structurels de I'insertion internationale.

Cela se produit al ors que le contexte intellectuel se transforme du fait de la contestation, sans cesse croissante depuis 2000, du paradigme du libre-échange (B ourguinat, 2005). La nouvelle équipe dirigeante, outre qu' elle est porteuse d' un volontarisme économique, y trouve une base pour dénoncer l'ouverture. Ce nouveau contexte intellectuel (domestique et systémique) coïncide avec une amélioration du contexte macroéconomique liée directement à I'accroissement des prix des hydrocarbures à partir du second semestre 1999. Le pays passant d'une situation de déficit de ressources financières (1986-1996) à une situation d'excédents (à partir de 2002), de nouvelles marges de manœuvre s'offrent aux dirigeants. Ceux-ci vont mettre en œuvre un ambitieux programme économique tiré par la dépense publique ( $M$ artin 2003 ; Talahite, Hammadache, 2011a).

L'amélioration de la compétitivité structurelle du pays, qui suppose dans la logique du décideur public, l'approfondissement de l'intégration internationale par l'ouverture aux capitaux productifs étrangers et la convergence institutionnelle vis-à-vis des partenaires, européens en particulier, devient prioritaire. Cette extension de la norme d' ouverture donnera lieu à une relance des négociations des accords internationaux, la signature de l'accord d'association avec I'UE (entré en vigueur en 2005) et l'adhésion à la Grande zone A rabe de libre-échange (engagée en 2004 et entrée en vigueur en 2009) ${ }^{10}$.

M ais en parallèle le discours critique vis-à-vis de l'ouverture s' amplifie, facilité en cela par la situation d'aisance financière du pays et par la faiblesse des gains après plus d'une décennie. Ce discours, auquel s'ajoutent ceux critiques du Président vis-à-vis des investisseurs étrangers (jeu des intérêts d'acteurs), émerge dans le sillage de la crise financière de 2007 (contexte macroéconomique) et du changement de gouvernement (juin 2008). Un consensus au sein de I'appareil étatique prend forme, s'appuyant sur le triple constat: i) de la faible diversification des exportations; ii) de l'accroissement de $30 \%$ des importations entre 2006 et 2008 et dont I'élasticité au PIB est passée de 0.3 en 2000 à 1.7 en 2008 pour une moyenne 1995-2008 de 2,57 et iii) de la quasi-absence d'IDE hors hydrocarbures (tableau 2 et figure 3) Bien que I'A Igérie ait ratifié 83 accords internationaux incitatifs à l'IDE dont 46 traités bilatéraux et 6 accords internationaux d'investissement (Unctad, 2011). Cela conduit à relativiser la thèse du manque d'incitations institutionnelles comme explication de la faible attractivité du pays (Djoufelkit, 2008).

\footnotetext{
${ }^{10}$ Convergeant avec l'objectif politique de sortir le pays de l'isolement international dans lequel I'ont plongé les années 1990, le dossier de l'adhésion à l'OM C devient prioritaire. Dans ce cadre, en 2001 la structure tarifaire est ramenée à 3 taux et le taux maximum est abaissé de 45 à $30 \%$. En termes nominaux, ce démantèlement fait passer la moyenne simple non pondérée de 23,4 à 17,5\%.
} 
Tableau 2 : Stock entrant de l'investissement direct étranger (milliards de \$ courants)

\begin{tabular}{|l|c|c|c|}
\cline { 2 - 4 } \multicolumn{1}{c|}{} & 1990 & 2000 & 2010 \\
\hline A Igérie & 1,6 & 3,5 & 19,5 \\
\hline M aroc & 3,0 & 8,9 & 31,4 \\
\hline Tunisie & 7,6 & 11,5 & 42,0 \\
\hline Egypte & 11,0 & 19,9 & 73,1 \\
\hline
\end{tabular}

Source : Cnuced, 2011.

Figure 3 : E ntrées nettes moyennes d'IDE 2000-2009 (pourcentage du PIB)

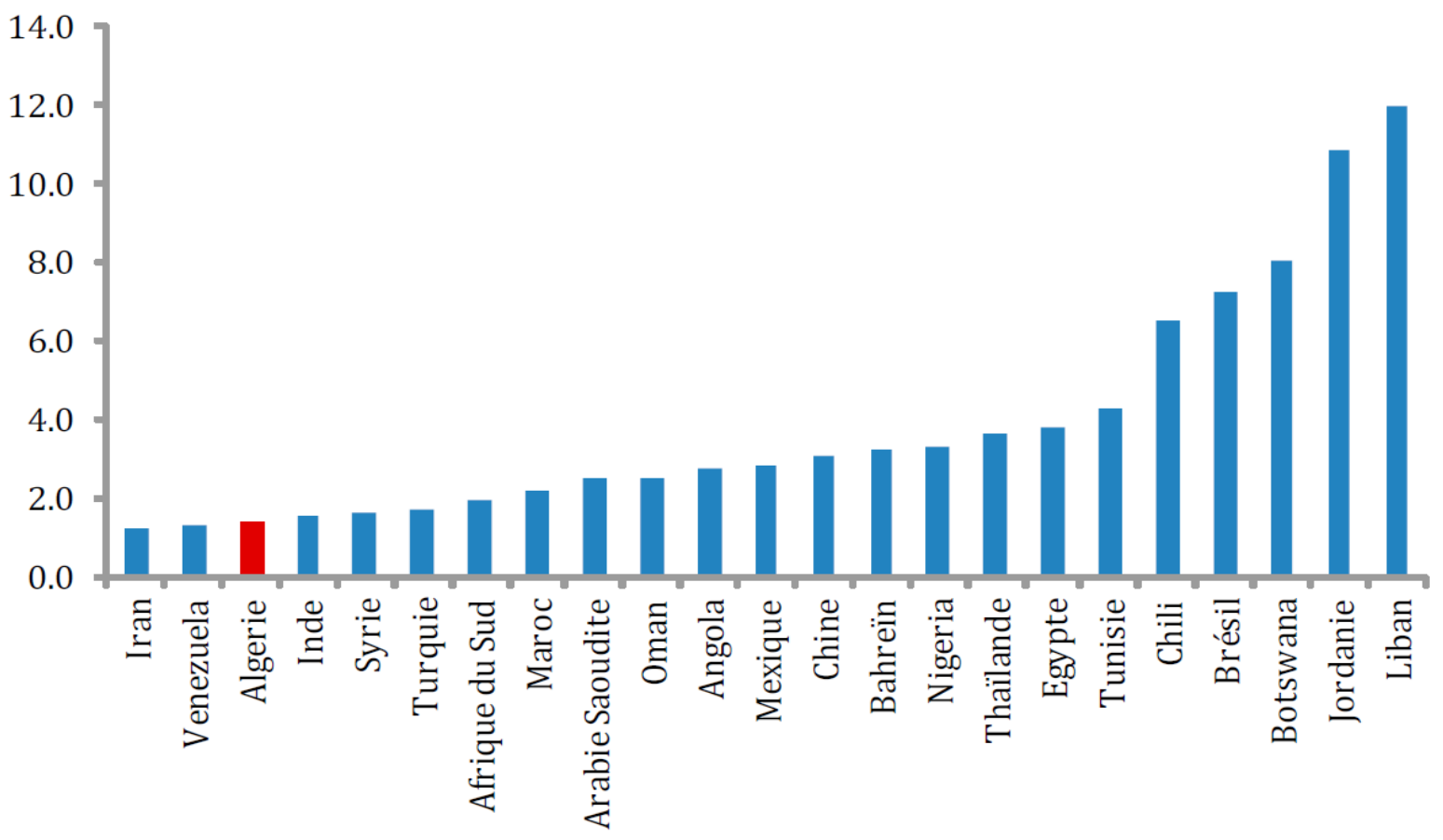

Source: B anque mondiale http://donnees.banquemondial e.org/catalogue/les-indicateurs-dudeveloppement-dans-le-monde, consultée le 21 juin 2011.

Ces éléments conduisent à un mouvement de re-étatisation de l'articulation nationalinternational au travers de dispositifs réglementaires contenus dans la loi de finances complémentaire de 2009 (LFC 2009). A cela s'ajoute une remise en cause des principaux processus d'ouverture dans lesquels l'Algérie est engagée (accession à l'OMC, accord d'association avec l'UE et GZALE). De plus, les autorités algériennes ont annoncé, début 
mars 2010, la non-ratification de tout nouvel accord de libre-échange tant que le dossier OM C ne sera pas clos.

Ce renoncement aux effets transformationnels de l'ouverture s' explique par l'amélioration du contexte macroéconomique sur laquelle se greffe l'évolution de la configuration des acteurs. Tout d'abord, les conflits de préférences et d'intérêts persistent au sein du SNEP, entraînant un manque de cohérence dans la stratégie d'ouverture ${ }^{11}$. La période 1999-2010 et in extenso les années 1985-2010 révèlent l'incapacité dans laquelle se trouve le décideur à développer une doctrine articulant de façon positive, et pas seulement en termes d'adaptation contrainte, développement et mondialisation. Ensuite, selon Mohsen-Finan (IFRI, 2010), la coalition libérale serait parvenue entre 2000 et 2011 à neutraliser les représentants du «complexe militaro-industriel ». Cela expliquerait, en partie, le processus de désindustrialisation (Benabdallah, 2009) que connaît le pays et la faible portée de la Nouvelle Stratégie Industrielle lancée en 2007. Enfin, l'échec de l'ouverture du secteur des hydrocarbures et de la réforme de la Sonatrach (2004-2005) montre que les coalitions profitant de l'exploitation de la rente ont consolidé leur position ( $M$ artinez, 2010). Ainsi l'économie politique de I'ouverture illustre la façon dont la coalition dominante reconfigure le champ politico-social en vue de maintenir son contrôle sur les dispositifs économiques d'exercice du pouvoir.

Ce type de comportement explique le paradoxe relevé par Sid-A hmed (2001) et Talha (2001) selon lequel l'ouverture extérieure n'a pas modifié les modalités dominantes de l'insertion internationale et $n^{\prime}$ a pas produit de diversification productive ou le constat de réformes incomplètes et inachevées (B ouhou, 2009). Les comportements d'adaptation n'ont pas suivi une logique productive permettant l'émergence de groupes socio-économiques industrialistes. Au contraire, I'adaptation visait le développement de nouveaux réseaux dont l'objectif prioritaire est d'obtenir un accès privilégié aux ressources financières dont dispose la coalition dominante.

\section{Conclusion}

Cette contribution est une tentative d'analyse de long terme de la stratégie d'ouverture de l'Algérie. Elle met en exergue trois facteurs : le contexte macroéconomique déterminé par la variable des prix internationaux des hydrocarbures, les compromis structurant le SNEP et le contexte intellectuel autorisant un mouvement de désétatisation du commerce extérieur. Elle permet d'établir les conclusions suivantes :

- Les analyses de la nouvelle économie institutionnelle et de la Banque mondiale (Edwards, 1987) qui résument l'échec d'une politique à l'absence d'un cadre institutionnel approprié dans un contexte d'asymétrie d'informations et de coûts de transaction élevés se révèle d'une faible portée explicative quant à la trajectoire d'ouverture de l'économie algérienne. En mettant en avant le conflit distributif lié aux effets transformationnels de l'ouverture, nous estimons que les arrangements institutionnels formels et informels du SNEP (réseaux institués, relation client-patron, rapports claniques, proximités familiales, etc.) conditionnent les contenus, modalités et formes de la politique d'ouverture. A ussi, la façon dont se constituent les compromis et se

\footnotetext{
${ }^{11}$ La diversification des exportations, objectif affiché du décideur politique en A lgérie, suppose, conformément au principe de réciprocité, une ouverture accrue du marché algérien et une perte de contrôle des procédures d'importations. II n' est pas sûr que certaines composantes du SNEP y soient disposées.
} 
neutralisent les intérêts contradictoires au sein du SNEP qui déterminent le rapport au monde de l'économie al gérienne ${ }^{12}$.

- Sur la période 1985-2010, il n'y a jamais eu une séquence temporelle où un contexte macroéconomique favorable aux réformes, une coalition élitaire relayée par des groupes socioéconomiques soutenant les réformes et un consensus politico-économique, ont été réunis. Cela explique que l'on assiste à des réformes sur les flux mais pas de structure qui voit se former, sur la période 1986-2011, un système clientéliste extraverti. La libéralisation commerciale survient dans des contextes de contractions des revenus d'hydrocarbures permettant une gestion du conflit distributif, l'Etat ayant recours à un autre type de rente à destination d'autres acteurs. Cela a pour effet de reconfigurer les groupes d'intérêt en concurrence pour l'accès aux ressources de l'E tat et de renouveler les procédures de légitimation au sein du SNEP.

- En plaçant l'Etat au centre des arbitrages relatifs à l'insertion internationale, l'économie politique complète la théorie de l'économie internationale et permettrait d'aborder les questions de faisabilité d'une réforme d'un régime rentier d'accumulation en économie ouverte et, dans une perspective plus normative, la nature des arrangements institutionnels à mettre en place, d'une part pour que l'ouverture produise pleinement ses effets transformationnels productifs, et, d'autre part pour que le conflit distributif qui lui est associé soit minimisé.

L'échange international est facteur de changement structurel et institutionnel à condition qu'il soit le prolongement d' une dynamique domestique, et le rapport au marché mondial est d'autant plus profitable qu'il s'articule à un marché national dynamique. De la sorte, ce sont les politiques nationales qui constituent les variables clés pour la croissance et le développement, et non les mesures de libéralisation de l'échange international.

\footnotetext{
12 Une étape ultérieure de la recherche consistera à établir une hiérarchie stricte entre ces déterminants et à spécifier des indicateurs précis permettant d'endogénéiser la périodisation proposée. II convient également d'affiner l'analyse des groupes d'acteurs influençant les choix de politique commerciale et la façon dont se construisent leurs préférences.
} 


\section{Bibliographie}

Abbas M., 2009, «L'accession à l'OMC : quelles stratégies pour quelle intégration à la mondialisation ? », Confluences M éditerranée, $n^{\circ} 71$, automne, pp. 101-118.

A ghrout A., Bougherira R., 2004, Algeria in Transition. Reforms and Development Prospects, London, Routledge.

Aïssaoui A., 2001, The Political Economy of Oil and Gas, Oxford, Oxford University Press.

Baldwin R., 1996, "The Political Economy of Trade Policy: Integrating the Perspectives of Economists and Political Scientists», in Feenstra R., Grossman G., Irwin D. (eds.), The Political Economy of Trade Policy: Papers in Honor of J agdish Bhagwati, Cambridge, M IT Press.

Baldwin R., 1989, «The Political Economy of Trade Policy », J ournal of Economic Perspective, 3, Fall, pp. 119-135.

B enabdallah Y ., 2009, Rente et désindustrialisation, Confluences M éditerranée, $n^{\circ} 71$, automne, pp. 85-100.

Benbitour A., 1992, L'expérience algérienne de développement. 1962-1991, Alger, ISGP Editions.

Benissad H., 1997, «Le plan d'ajustement structurel », Confluences Méditerranée, $n^{\circ} 23$, A utomne, pp. 107-118.

B enissad, H., 1991, La réforme économique en Algérie ou l'indicible ajustement structurel, $2^{\text {e }}$ édition mise à jour, OPU, Alger.

Bouhou K., 2009, "L'Algérie des réformes économiques : un gout d'inachevé », Politique étrangère, $n^{\circ} 2$, pp. 323-335.

Bourguinat $H ., 2005$, «Le libre-échange : un paradigme en situation d'inconfort », Revue d'économie politique, $\mathrm{V}$ ol. $115, \mathrm{n}^{\circ}$ 5, pp. 531-543.

Bouyacoub A., 1997, "L'économie algérienne et le programme d'ajustement structurel », Confluences Méditerranée, $n^{\circ} 21$, A utomne, pp. 77-85.

B rahimi A., 1991, L'économie algérienne, OPU, Alger.

Cassing J., 1991, «A Theory of Changes in Trade Policy Regimes », in Hillman A., M arkets and Politicians: Politicized Economic Choice, Boston, K luwer A cademic.

Chang H. J., 1993, "The Political Economy of Industrial Policy in Korea », Cambridge J ournal of Economics, vol. 17, $\mathrm{n}^{\circ} 2$, pp. 131-157.

Corm, G. (1993), « La réforme économique algérienne : une réforme mal aimée ? », MaghrebMachrek, $n^{\circ} 139$, janvier-mars, pp. 9-27.

Dahmani A., 1999, L'Algérie à l'épreuve. Economie politique des réformes 1980-1997, Paris, L'Harmattan.

Destler I., Odell J., 1987, Anti-Protection: Changing Forces in U nited States Trade Politics, Policy A nalyses in International Economics, $n^{\circ} 21$, Washington, Institute for International Economics.

Djoufelkit H., 2008, «Rente, développement du secteur productif et croissance en Algérie », AFD, document de travail, $n^{\circ} 64$, juin.

Drazen A., Grilli V ., 1993, "The B enefit of Crisis for Economic R eforms », American Economic Review, vol. 83, n³, pp. 598-607.

Ecrement M., 1986, Indépendance politique et libération économique. Une quart de siècle du développement de l'Algérie, 1962-1985, ENAP/PUG, Alger/Grenoble.

Edwards S., 1987, "Trade Liberalization Reforms and the World Bank », American Economic Review, vol. 87, n², pp. 43-48.

Fernandez R., Rodrik D., 1991, "Resistance to Reform: Status Quo Bias in the Presence of Individual-Specific Uncertainty », American E conomic Review, vol. 81, n 5, pp. 1146-1155. 
Garret G., Lange P., 1996, «Internationalization, Institutions and Political Change », in K eohane R. O., M ilner H. V. (eds.), Internationalization and Domestic Politics, N ew-Y ork, Cambridge University Press.

Gilpin R., 1987, The Political Economy of International Relations, Princeton, Princeton University Press.

Goldstein J., 1988, «Ideas, Institutions and American Trade Policy», International Organization, vol. 42, $n^{\circ} 2$, Winter, pp 179- 217.

Goldstein J., Keohane R. (eds.), 1993, Ideas and Foreign Policy: Beliefs, Institutions, and Political Change, Ithaca, Cornell University Press,

Goumeziane S., 1994, «L'incontournable libéralisation », Confluences M éditerranée, nº 11, été, pp. 41-54.

Gourevitch P., 1986, Politics in Hard Times. Comparative Responses to International Economic Crises, London, Cornell U niversity Press.

Hall P. A., 1997, "The Role of Interests, Institutions, and Ideas in the Comparative Political Economy of the Industrialized Nations», in Lichbach M. A., Zuckerman A. S. (dirs), Comparative Politics: Rationality, Culture, and Structure, N ew-Y ork, Cambridge University Press, pp. 174-207.

Henni A ., 1991, Economie de l'Algérie indépendante, A lger, Enag éditions.

Henry C. (2005), "Algeria's A gonies: Oil Rent Effects in a Bunker State », in Bonner M., Reif M. \& Tessler M . (2005), Islam, Democracy and the State in Algeria. Lessons for the Western Mediterranean and Beyond, London, Routledge, pp. 68-81.

Hillman A., 1989, The Political Economy of Protection, New-Y ork, Harwood A cademic.

IFRI., 2010, Ramses 2011, Paris, Dunod.

IM F, 1998, Algeria: Stabilization and Transition to Market, IM F Occasional Paper, $n^{\circ} 165$, IM F, Washington.

IMF, Algeria Country Report, divers numéros, Washington, IM F. Consultables à I'adresse suivante: http://www.imf.org/external/country/dza/index.htm

K han M. 1995, "State Failure in Weak States: A Critique of $\mathrm{New}$ Institutionalist Explanations », in Harriss J., Hunter J., Lewis C. (eds.), The New Institutional Economics and Third World D evelopment, London, Routledge.

Krueger A. O., 1993, The Political Economy of Policy Reform in Developing Countries, Cambridge, M IT.

Luciani G., 1987, «Allocation vs. Production States: A Theoretical Framework », in Beblawi H., Luciani G. (dir.), The Rentier State, London, Croom Helm, pp. 63-82.

M artin I. 2003, "A Igeria's Political Economy (1999-2002) : An Economic Solution to the Crisis », The J ournal of North African Studies, V ol. 8, n² 2, Summer, pp. 34-72.

Martinez L., 2010, "Algérie: les illusions de la richesse pétrolière », Les Etudes du CERI, $\mathrm{n}^{\circ} 168$.

Nelson D., 1999, "The Political Economy of Trade Policy Reform: Social Complexity and Methodological Pluralism», Journal of international Trade and Economic Development, vol. $8, n^{\circ} 1$, pp. 3-26.

North D., W allis J., W eingast H., 2010, Violence et ordres sociaux, Paris, Gallimard.

Rodrik D., 1995a, «Political Economy of Trade Policy », in Grossman G., Rogoff K. (dirs.), Handbook of International E conomics, V olume 3 , N orth Holland, A msterdam.

Rodrik D., 1995b, "The Rush to Free Trade in the Developing World: Why So Late? Why Now? Will It Last? », in Haggard S., Webb S. B. (eds.), Voting for Reform : Democracy, Political Liberalization, and Economic Adjustment, N ew Y ork, Oxford University Press.

Rogowski R., 1989, Commerce and Coalitions : How Trade Affects Domestic Political Alignments, Princeton, Princeton University Press. 
Schwartz H. M., 1994, States versus M arkets - H istory, Geography and the Development of the International Political Economy, New-Y ork, St M artin's Press.

Seers D. (1964), "The Mechanism of an Open Petroleum Economy », Social and Economic Studies, vol. $13, \mathrm{n}^{\circ} 14$.

Sid A hmed A. (2004), Le développement asiatique : quels enseignements pour les économies arabes ? Eléments de stratégie de développement : le cas algérien, Paris, Publisud.

Sikkink K., 1991, Ideas and Institutions: Developmentalism in Brazil, and Argentina, NewY ork, Cornell U niversity Press.

Smith A. (2000), Recherches sur la nature et les causes de la richesse des nations, (1 ${ }^{\text {ère }}$ éd. 1776), Paris, Economica.

Talahite F., H ammadache A ., 2011a, «L'économie al gérienne. D' une crise à I' autre », MaghrebMachrek, n²06, pp.101-123.

Talahite F., Hammadache A., 2011b, «L'économie algérienne dans le contexte de la crise financière internationale», Journée d'études EDDEN-CEPN «L'Algérie aux défis de la globalisation », Grenoble, 8 février.

Talha L. (2001), « L'économie algérienne au milieu du gué : le régime rentier à l'épreuve de la transition institutionnelle », in Mahiou A., Henry J-R. (dirs.), Où va l'Algérie?, Paris, Karthala.

Unctad, 2011, World Investment Report, Geneva, U nited N ations.

Werenfels I., 2007, Managing Instability: Elites and Political Change in Algeria, London, Routledge.

W orld B ank, 2011, World Trade Indicators 2009/10, W ashington, W orld B ank. Disponible à http://web.worldbank.org/W BSITE/EXTER NA L/TOPICS/TRA DE/0 „, contentM DK :2242195 0 pagePK :148956 piPK :216618 theSitePK :239071,00.html 